\title{
A Simple Contact Mechanics Model for Highly Strained Aqueous Surface Gels
}

\author{
A. L. Chau ${ }^{1} \cdot$ M. K. Cavanaugh ${ }^{2} \cdot$ Y.-T. Chen ${ }^{2} \cdot$ A. A. Pitenis ${ }^{1}$ (D)
}

Received: 6 October 2020 / Accepted: 1 February 2021 / Published Online: 16 February 2021

(c) The Author(s) 2021

\begin{abstract}
Background Soft, biological, and bio-inspired materials are often compositionally heterogeneous and structurally anisotropic, and they frequently feature graded or layered organizations. This design complexity enables exceptional ranges in properties and performance yet complicates a fundamental understanding of the contact mechanics. Recent studies of soft gel layers have relied on Hertzian or Winkler foundation ("bed-of-springs") models to characterize the mechanics but have found neither satisfactory.

Objective The contact mechanics of soft gel layers are not yet fully understood. The aim of this work is to develop a simple contact mechanics model tailored for compositionally-graded materials with soft surface layers under high strains and deformations.

Methods Concepts from polymer physics, fluid draining, and Winkler foundation mechanics are combined to develop a simple contact mechanics model which relates the applied normal force to the probe radius of curvature, elastic modulus, and thickness of soft surface layers subjected to high strains.

Results This simple model was evaluated with two examples of graded surface gel layers spanning multiple length-scales, including commercially available contact lenses and stratified hydrogels. The model captures the nonlinear contact mechanics of highly strained soft aqueous gel layers more closely than either Hertz or Winkler foundation theory while simultaneously enabling a prediction for the thickness of the surface gel layer.
\end{abstract}

Conclusion These results indicate that this simple model can adequately characterize the contact mechanics of highly strained soft aqueous gel layers.

Keywords Aqueous gels · Contact mechanics · Biotribology

\section{Nomenclature}

$\Pi \quad$ Osmotic pressure

E Elastic modulus

$k_{B} \quad$ Boltzmann constant

$T$ Temperature

$\xi \quad$ Polymer mesh size

c Polymer concentration

$c_{o} \quad$ Initial polymer concentration

$c_{d} \quad$ Polymer concentration after draining

$R \quad$ Probe radius of curvature

$z \quad$ Indentation depth

A. A. Pitenis

apitenis@ucsb.edu

1 Materials Department, University of California, Santa Barbara, Santa Barbara, CA 93106, USA

2 Department of Chemical Engineering, University of California, Santa Barbara, Santa Barbara, CA 93106, USA $z_{o} \quad$ Indentation depth at maximum pressure

$s \quad$ Distance from the probe apex

$s_{\max }$ Contact area radius

$t \quad$ Surface gel layer thickness

A Contact area

$P \quad$ Contact pressure

\section{Introduction}

Gels protect all aqueous sliding surfaces in biology (e.g., ocular tear films, cartilaginous joints, mucosal surfaces), yet their fundamental mechanics remain elusive. Over the past few decades, increasingly sophisticated experimental methods have been developed to characterize the mechanics of biological and compliant materials $[1,2]$ and mimic their complex hierarchical structures. [3] Arguably two of the most common contact mechanics models deployed to 
analyze soft material systems are Hertz and Winkler. [4, 5] However, there are several underlying assumptions that preclude the use of Hertzian contact mechanics in biological material systems, notably those of small deformations and material homogeneity. [6] Biological samples are often subjected to very large deformations and are highly heterogeneous, especially at smaller lengthscales, [7] due to their inherent anisotropy and extreme softness. Winkler foundation theory similarly falls short in applications involving thick and structurally-heterogeneous biological samples. Significant efforts have been put forth by many groups to characterize the poroelastic effects of soft gels during indentation [8-16], from the undrained limit (instantaneous response) through the transient response to the drained limit (equilibrium response). With few exceptions, most contact mechanics models are used to investigate relatively narrow ranges of soft gel indentation responses and are limited to low strains and deformations. Here we probe the drained limit of poroelasticity for aqueous surface gel layers by proposing a simple analytical contact mechanics model that combines Winkler foundation mechanics [17-20] and considerations of fluid draining. [21] We evaluate this model using nanoindentation data of water gradient contact lenses [22] and stratified hydrogels [23] and compare the model against results obtained using Hertz, Winkler, and poroelastic $[10,11]$ contact mechanics models.

\section{Model Derivation}

The basis for this contact mechanics model combines concepts of poroelasticity, [15] draining, [21] and Winkler foundation mechanics, [17] which is often applied to rigid thin films atop soft substrates. $[17,20]$ This model is designed to analyze the mechanics of soft aqueous gel layers, from biomedical devices to synthetic hydrogels. Soft aqueous gels under persistent loads will initially undergo diffusion-driven dynamic polymer network re-arrangement. [24] As demonstrated by the Angelini group, hydrogels do not relinquish water (drain) until the applied contact pressure exceeds the osmotic pressure of the hydrogel network. [24, 25] According to the scaling principles determined by de Gennes, [26] osmotic pressure, $\Pi$, scales with the elastic modulus, $E$, as shown in equation (1):

$\Pi \sim \mathrm{E} \sim \frac{k_{B} T}{\xi^{3}} \sim c^{\frac{9}{4}}$

where $k_{B}$ is the Boltzmann constant, $T$ is temperature, $\xi$ is mesh size, and $c$ is polymer concentration. Utilizing geometry and the small angle approximation, the contact area radius is estimated as $s_{\max }=\sqrt{2 z_{0} R}$ where $z_{o}$ is the indentation depth and $R$ is the radius of curvature of the probe (Fig. 1(a)).

With a spherical probe geometry, the applied pressure is distributed across the contact area as described by $d F=A P=2 \pi s d s \cdot P_{z}$ where $P_{z}$ is the applied pressure and $s$ is the radial distance from the center of the spherical probe, which is the location of maximum pressure. To obtain the total force within the contact area, $d F$ is integrated from the center of the probe, defined as $s=0$, to $s=s_{\max }$, leading to:

$F=2 \pi \int_{0}^{s_{\max }} s P_{z} d s$

Once the applied load surpasses the osmotic pressure, draining will occur as fluid flows away from the contact zone. This exudation of fluid leads to an increase in polymer concentration, as shown schematically (Fig. 1(b), (c)). To account for this increase in concentration under compressive load, the polymer concentration can be redefined as equation (3):

$c_{d}=\frac{V_{\text {polymer }}}{(t-z) d A}=\frac{c_{o} t d A}{(t-z) d A}=\frac{c_{o} t}{t-z}$

where $c_{o}$ is the initial polymer concentration of the hydrogel and $c_{d}$ is the concentration of the hydrogel after draining. Pressure, $P_{z}$, scales with elastic modulus, $E$, and polymer concentration, as shown in equation (1). With the addition of a scaling coefficient, $\lambda$, pressure is defined to equate
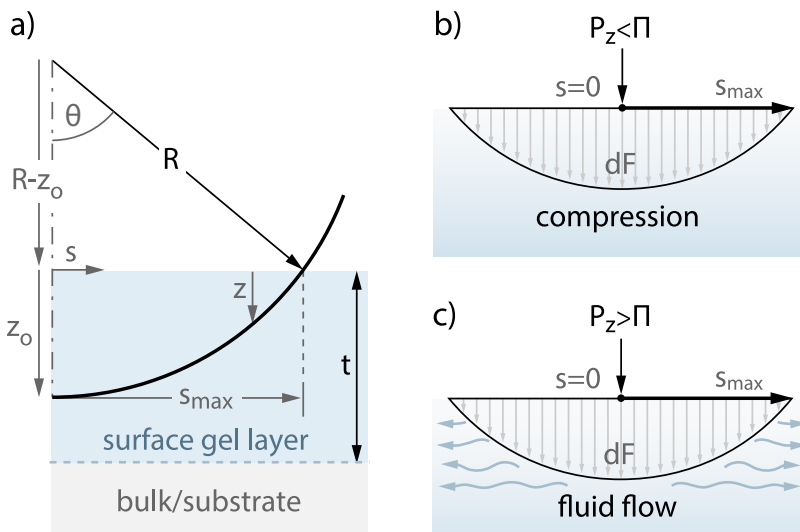

c)

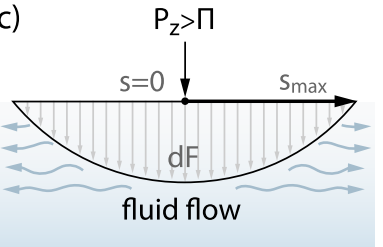

Fig. 1 Schematic of probe and sample geometry. (a) Surface gels (light blue) of thickness $t$ may be layered atop bulk material or rigid substrates (light gray). b, c) Illustrations of indentations at contact pressures exceeding the osmotic pressure of the surface gel layer. Cross-sections of spherical probe indenting surface gel layer with applied pressure $P_{z}$ calculated from differential normal forces $d F$ spread throughout the contact from the center, $s=0$, to the edge of contact, $s=s_{\max }$. Both (b) polymer compression and (c) fluid flow (draining) contribute to increased subsurface polymer concentration with increasing indentation depth 
polymer concentration as $P_{z}=\lambda c_{d}^{\frac{9}{4}}=\lambda\left(\frac{c_{o} t}{t-z}\right)^{\frac{9}{4}}$. The parameter $z$ can be redefined in terms of indentation depth at maximum pressure $\left(z_{0}\right)$, probe radius of curvature $(R)$, and distance from the center of the probe $(s)$ as $z=z_{o}-\frac{s^{2}}{2 R}$. Thus equation (2) can be rewritten as:

$F=2 \pi \int_{0}^{s_{\max }} s \lambda\left(\frac{c_{o} t}{t-z}\right)^{\frac{9}{4}} d s=2 \pi \int_{0}^{\sqrt{2 z_{o} R}} s \lambda\left(\frac{c_{o} t}{t-z_{o}+\frac{s^{2}}{2 R}}\right)^{\frac{9}{4}} d s$

Integrating the equation leads to equation (5):

$F=\frac{8 \pi R \lambda t^{\frac{9}{4}} c_{o}^{\frac{9}{4}}}{5\left(t-z_{o}\right)}-\frac{8 \pi R \lambda t^{2} c_{o}^{\frac{9}{4}}}{5\left(t-z_{o}\right)}+\frac{8 \pi R \lambda t c_{o}^{\frac{9}{4}} z_{o}}{5\left(t-z_{o}\right)}$

where $\lambda=\frac{E}{9 / 4}$, allowing Eq. 5 to be rearranged and simplified as:

$F=\frac{8 \pi R t E}{5}\left[\left(\frac{t}{t-z_{o}}\right)^{\frac{5}{4}}-1\right]$

From the model, the force is dependent on the surface gel layer thickness $(t)$, indentation depth at maximum pressure $\left(z_{o}\right)$, probe radius of curvature $(R)$, and elastic modulus $(E)$.

\section{Results and Discussion}

Two examples of surface gel layers were selected from the literature to evaluate the efficacy of this model compared to Hertz, Winkler, and poroelastic models. The first example was a delefilcon A contact lens, which had an average thickness of about $100 \mu \mathrm{m}$ and a highly hydrated (> 80\% water content) covalently-crosslinked, silicone-free surface gel layer (approximately $5 \mu \mathrm{m}$ thick) attached to a core silicone hydrogel material (33\% water content). Dunn et al. performed nanoindentation experiments using a $5 \mu \mathrm{m}$ diameter silica microsphere loaded against a delefilcon A lens equilibrated in borate-buffered saline at room temperature. [22] The indentation speed of the plasmacleaned, piezoelectric-driven probe was $1 \mu \mathrm{m} \mathrm{s}^{-1}$ and the maximum indentation depth was about $400 \mathrm{~nm}$. The authors analyzed a portion of the force-displacement curve using Hertzian contact mechanics for indentation depths within the first $200 \mathrm{~nm}$ of the surface and estimated an elastic modulus of about $25 \mathrm{kPa}$. Beyond this indentation depth, the authors acknowledged that the response deviated from the Hertzian relationship. However, the model herein can capture this higher strain regime and was applied to the entire approach curve using a probe radius of curvature of $R=2.5 \mu \mathrm{m}$ and Poisson's ratio of $v=0.5$. An elastic modulus of $E=3.2 \mathrm{kPa}$ and a surface gel layer thickness of $t=500 \mathrm{~nm}$ was estimated, indicating that a maximum strain of $86 \%$ was reached during the experiment (Section S2, Supplementary Materials). These values generally agree with the literature $[22,27]$ and suggest that the ultrastructure of the contact lens' compositionally-graded surface may be even softer across the 400-nm region tested than originally predicted. Based on the model, the soft surface gel layer likely drained during compression and strain-stiffened (Fig. S1, Supplementary Materials). [28-30] The model herein tracks the strain-stiffening behavior much more closely than the Hertz, Winkler foundation, or poroelastic contact mechanics models (Section S2-3, Supplementary Materials).

The second example is a polyacrylamide hydrogel with intentional gradients in polymer concentration from casting against a hydrophobic (polystyrene) surface. [23, 31-33] The ability to create superlubricious hydrogel surfaces from changing the surface energy of the molding surface [31, 34] or removing the molding surface altogether [35] has been known for decades, yet the precise structures that arise from these processes are just beginning to be understood. [32, 33] The Spencer group recently probed the top $10 \mu \mathrm{m}$ of a polystyrene-molded hydrogel with a silica microsphere (14 $\mu \mathrm{m}$ radius) at a rate of $1 \mu \mathrm{m} \mathrm{s} \mathrm{s}^{-1}$ using atomic force microscopy (AFM, MFP-3D ${ }^{\mathrm{TM}}$, Asylum Research, Santa Barbara, USA). [23] Simič et al. analyzed a portion of the force-displacement curves with Hertzian contact mechanics and predicted an elastic modulus of less than $0.1 \mathrm{kPa}$ from the initial $1 \mu \mathrm{m}$ of indentation depth. [23] Using a Poisson's ratio of $v=0.5$, Hertzian, Winkler foundation, and poroelastic models were fit to the entire approach curve (Fig. 2(b)) and compared with the model developed herein. The simple contact mechanics model was the most effective in capturing the full mechanical response under load, particularly at large deformations and high strains (>60\%) (Fig. S2(b), Supplementary Materials). The model estimated an elastic modulus of $E=26 \mathrm{~Pa}$, which agrees with the literature, [23] and predicted a surface gel layer thickness of $t=12 \mu \mathrm{m}$ (compared to an overall sample thickness of 3-4 mm). This estimation aligns well with the range of surface gel layer thickness offered by Simič et al. of 10-20 $\mu \mathrm{m}$. [23].

One of the primary limitations of this model is that the stratified surface gel layer is difficult to discern if it is significantly smaller than the indentation depth of the sample. The model is most effective when the surface gel layer is slightly larger than the indentation depth of the experiment. Thus, incrementally deeper indentations on a surface gel layer may be needed to estimate the approximate thickness of the surface gel layer. The model is also limited by the assumption that draining occurs via indentation using a sphere-on-flat contact 

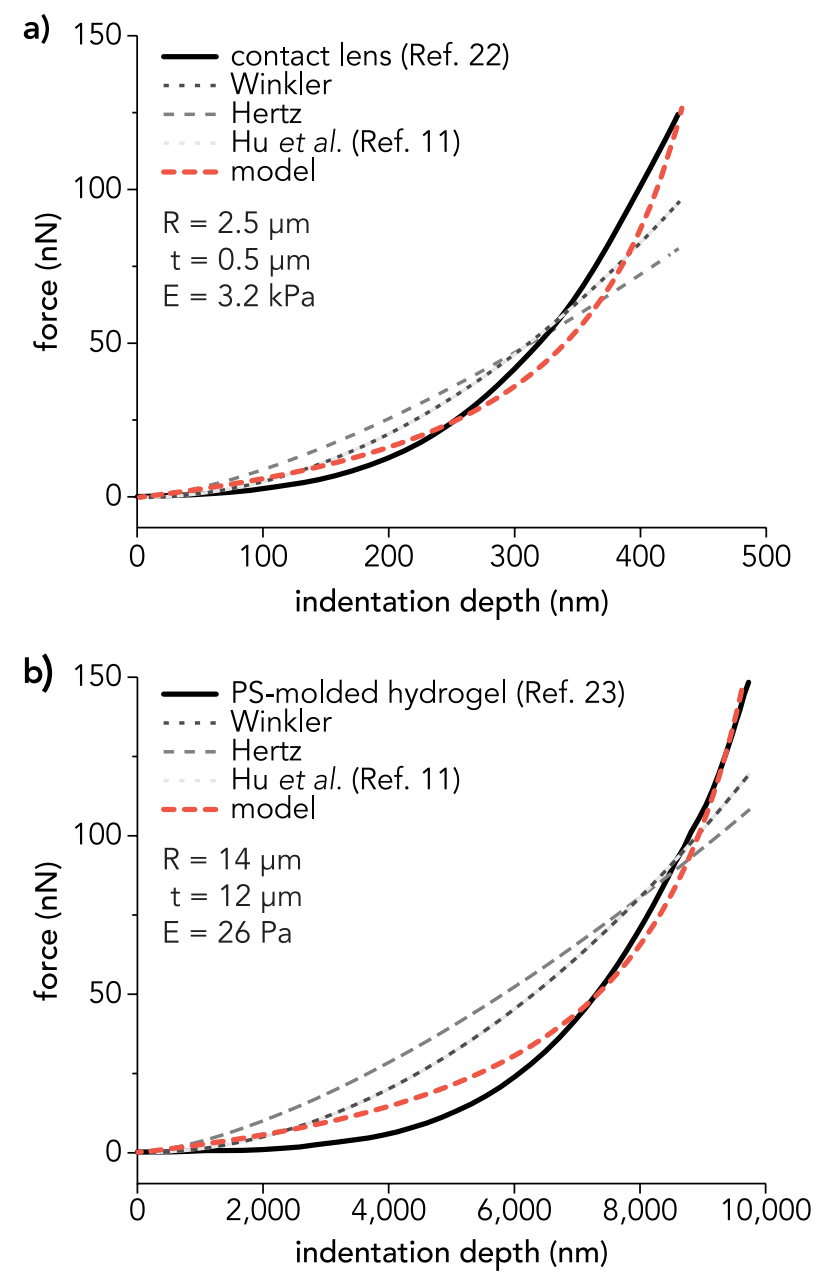

Fig. 2 Force-displacement data for two aqueous surface gel systems. Nanoindentation data (solid black line) of (a) water gradient contact lens [22] and (b) polystyrene-molded polyacrylamide hydrogel surface [23] fit with the Winkler foundation model (dotted dark gray line), Hertz model (dashed dark gray line), and poroelastic model put forth by $\mathrm{Hu}$ et al. [11] (light gray dashed line), compared with the model presented herein (dashed red line) with key parameters $R, t$, and $E$ provided. The Winkler model and poroelastic model proposed by $\mathrm{Hu}$ et al. almost perfectly overlap at higher indentation depths but deviate at very low indentation depths (Figs. S2 and S3, Supplementary Materials)

geometry, and future work will expand the model to account for parallel plate compression testing. Another limitation of the model is that it is based on polymer scaling relationships of flexible chains swollen in good solutions. [26] Future work will expand the model to accommodate complex, structurallygraded biological materials and tissues with major structural components that may be composed of semiflexible or rigid polymer networks and that may be swollen in proteinaceous solutions.

\section{Conclusions}

The simple contact mechanics model presented herein is based on a Winkler foundation model modified for increased polymer concentration following large deformations and high strains $(>60 \%)$. The model uses normal force and probe radius of curvature as inputs and can be used to solve for the elastic modulus and probable surface gel layer thickness. Compared to Hertzian, Winkler foundation, and poroelastic models, this model can capture a greater portion of the force-displacement curve, particularly at the drained limit (equilibrium response) and may provide a simple yet adequate route to quickly estimate the elastic modulus and surface gel layer thickness of biological and synthetic aqueous gels. The model may enable fundamental mechanics studies of more complex gradient gel structures.

Supplementary Information The online version contains supplementary material available at https://doi.org/10.1007/s11340-021-00699-5.

Acknowledgements This work was supported by the National Science Foundation (NSF) Materials Research Science and Engineering Center (MRSEC) at UC Santa Barbara through DMR-1720256 (IRG-3). The UCSB MRSEC is a member of the Materials Research Facilities Network (www.mrfn.org). A.L.C. acknowledges support of the National Science Foundation Graduate Research Fellowship Program under Grant No. 1650114. The authors acknowledge support from the California NanoSystems Institute (CNSI) Challenge Grant program.

\section{Declarations}

Conflict of Interest The authors have no conflicts of interest to declare.

Open Access This article is licensed under a Creative Commons Attribution 4.0 International License, which permits use, sharing, adaptation, distribution and reproduction in any medium or format, as long as you give appropriate credit to the original author(s) and the source, provide a link to the Creative Commons licence, and indicate if changes were made. The images or other third party material in this article are included in the article's Creative Commons licence, unless indicated otherwise in a credit line to the material. If material is not included in the article's Creative Commons licence and your intended use is not permitted by statutory regulation or exceeds the permitted use, you will need to obtain permission directly from the copyright holder. To view a copy of this licence, visit http://creativecommons.org/licenses/by/4.0/.

\section{References}

1. Franck C, Hong S, Maskarinec SA, Tirrell DA, Ravichandran G (2007) Three-dimensional full-field measurements of large deformations in soft materials using confocal microscopy and digital volume correlation. Exp Mech 47:427-438

2. Evans DW, Vavalle NA, DeVita R, Rajagopalan P, Sparks JL (2013) Nano-indentation device for investigating the mechanics of compliant materials. Exp Mech 53:217-229 
3. Lee D, Golden K, Rahman MM, Moran A, Gonzalez B, Ryu S (2019) Fabrication of hydrogels with a stiffness gradient using limited mixing in the hele-shaw geometry. Exp Mech 59:1249-1259

4. Garcia M, Schulze KD, O'Bryan CS, Bhattacharjee T, Sawyer WG, Angelini TE (2017) Eliminating the surface location from soft matter contact mechanics measurements. Tribol Mater Surfaces Interfaces 11:187-192

5. Garcia M, Angelini TE (2019) A method for eliminating the need to know when contact is made with soft surfaces: Data processing and error analysis. Biotribology 20:100109

6. Akhtar R, Sherratt MJ, Cruickshank JK, Derby B (2011) Characterizing the elastic properties of tissues. Mater Today 14:96-105

7. Proestaki M, Ogren A, Burkel B, Notbohm J (2019) Modulus of fibrous collagen at the length scale of a cell. Exp Mech 59:1323-1334

8. Galli M, Oyen ML (2008) Spherical indentation of a finite poroelastic coating. Appl Phys Lett 93:031911

9. Cai S, Hu Y, Zhao X, Suo Z (2010) Poroelasticity of a covalently crosslinked alginate hydrogel under compression. J Appl Phys 108:113514

10. Long R, Hall MS, Wu M, Hui C-Y (2011) Effects of Gel Thickness on Microscopic Indentation Measurements of Gel Modulus. Biophys J 101:643-650

11. Hu Y, Chan EP, Vlassak JJ, Suo Z (2011) Poroelastic relaxation indentation of thin layers of gels. J Appl Phys 110:086103

12. Kalcioglu ZI, Mahmoodian R, Hu Y, Suo Z, Van Vliet KJ (2012) From macro- to microscale poroelastic characterization of polymeric hydrogels via indentation. Soft Matter 8:3393

13. Bouklas N, Landis CM, Huang R (2015) A nonlinear, transient finite element method for coupled solvent diffusion and large deformation of hydrogels. J Mech Phys Solids 79:21-43

14. Delavoipière J, Tran Y, Verneuil E, Chateauminois A (2016) Poroelastic indentation of mechanically confined hydrogel layers. Soft Matter 12:8049-8058

15. Reale ER, Dunn AC (2017) Poroelasticity-driven lubrication in hydrogel interfaces. Soft Matter 13:428-435

16. Degen GD, Chen Y-T, Chau AL, Månsson LK, Pitenis AA (2020) Poroelasticity of highly confined hydrogel films measured with a surface forces apparatus. Soft Matter 16:8096-8100

17. Winkler E (1867) Die Lehre von der Elasticität und Festigkeit mit besonderer Rücksicht auf ihre Anwendungen in der Technik. Vol. 1, Prag: Theil. H Dominicus

18. Rennie AC, Dickrell PL, Sawyer WG (2005) Friction coefficient of soft contact lenses: measurements and modeling. Tribol Lett 18:499-504

19. Hill IJ, Sawyer WG (2010) Energy, adhesion, and the elastic foundation. Tribol Lett 37:453-461
20. Dillard DA, Mukherjee B, Karnal P, Batra RC, Frechette J (2018) A review of Winkler's foundation and its profound influence on adhesion and soft matter applications. Soft Matter 14:3669-3683

21. Pitenis AA, Sawyer WG (2018) Lubricity of high water content aqueous gels. Tribol Lett 66:113

22. Dunn AC, Urueña JM, Huo Y, Perry SS, Angelini TE, Sawyer WG (2013) Lubricity of surface hydrogel layers. Tribol Lett 49:371-378

23. Simič R, Yetkin M, Zhang K, Spencer ND (2020) Importance of hydration and surface structure for friction of acrylamide hydrogels. Tribol Lett 68:64

24. Schulze KD, Hart SM, Marshall SL, O'Bryan CS, Urueña JM, Pitenis AA, Sawyer WG, Angelini TE (2017) Polymer osmotic pressure in hydrogel contact mechanics. Biotribology 11:3-7

25. Bhattacharyya A, O'Bryan C, Ni Y, Morley CD, Taylor CR, Angelini TE (2020) Hydrogel compression and polymer osmotic pressure. Biotribology 22:100125

26. De Gennes PG (1979) Scaling concepts in polymer physics. Cornell University Press

27. Urueña JM, Pitenis AA, Nixon RM, Schulze KD, Angelini TE, Sawyer WG (2015) Mesh size control of polymer fluctuation lubrication in gemini hydrogels. Biotribology 1-2:24-29

28. Cao A, Dickrell PL, Sawyer WG, Ghasemi-Nejhad MN, Ajayan PM (2005) Super-compressible foamlike carbon nanotube films. Science 310:1307-1310

29. Erk KA, Henderson KJ, Shull KR (2010) Strain stiffening in synthetic and biopolymer networks. Biomacromol 11:1358-1363

30. Jaspers M, Dennison M, Mabesoone MFJ, MacKintosh FC, Rowan AE, Kouwer PHJ (2014) Ultra-responsive soft matter from strain-stiffening hydrogels. Nat Commun 5:5808

31. Meier YA, Zhang K, Spencer ND, Simic R (2019) Linking friction and surface properties of hydrogels molded against materials of different surface energies. Langmuir 35:15805-15812

32. Gombert Y, Simič R, Roncoroni F, Dübner M, Geue T, Spencer ND (2019) Structuring hydrogel surfaces for tribology. Adv Mater Interfaces 6:1901320

33. Gombert Y, Roncoroni F, Sanchez-Ferrer A, Spencer ND (2020) The hierarchical bulk molecular structure of poly(acrylamide) hydrogels: beyond the fishing net. Soft Matter

34. Gong JP, Kurokawa T, Narita T, Kagata G, Osada Y, Nishimura G, Kinjo M (2001) Synthesis of hydrogels with extremely low surface friction. J Am Chem Soc 123:5582-5583

35. Pitenis AA, Urueña JM, Cooper AC, Angelini TE, Sawyer WG (2016) Superlubricity in gemini hydrogels. J Tribol 138

Publisher's Note Springer Nature remains neutral with regard to jurisdictional claims in published maps and institutional affiliations. 